\title{
Impact of Direct Acting Antiviral Drugs (DAADs) on Cognitive function among Hepatitis C Virus Infected Patients \\ Hassan Salah Eldin Hamdy ${ }^{1}$, Mohamed Salah ELdin Abd El Hamid ${ }^{1}$, Mohamed Ahmed Shafik², Enass Mohamed Ahmed Mohamed Soliman ${ }^{*}$ \\ ${ }^{1}$ Department of Tropical Medicine, ${ }^{2}$ Department of Neurology Medicine, Faculty of Medicine, Ain Shams University *Corresponding Author: Enass Mohamed Ahmed Mohamed Soliman, Phone No.: (+2) 01007821298, E-mail: coatedbaby91@ gmail.com
}

\begin{abstract}
Background: chronic hepatitis $\mathrm{C}$ virus (HCV) is a major cause of liver-related morbidity and mortality. Successful antiviral therapy with sustained viral clearance is associated with improved quality of life, and reduced risk of liver complications such as cirrhosis and hepatocellular carcinoma. Aim of the Work: to evaluate the impact of $\mathrm{HCV}$ eradication using direct acting antiviral drugs (DAADs) on cognitive function among hepatitis c virus infected patients. Patients and Methods: this study was conducted at Viral Hepatitis Unit of Dar El Shefaa Hospital (one of the centers of national committee for control of viral hepatitis) and MASRI (Faculty of Medicine Ain-Shams Research institute) unit at El-Demerdash Hospital. Data were prospectively collected using MoCA, PHQ-9 and PSQI scales to assess the cognitive function of the treated patients with the newly used DAADs before the start of treatment, during treatment and at 12 weeks after the end of treatment, and the laboratory data were collected from the files of the patients in the previously mentioned centers. Results: results of the current study as regarding the analytical part of the study showed that: Pre treatment: slightly cognitive impairment was found with mild level of depression and sleep disturbance with MoCA test Mean \pm SD $23.02 \pm 4.96$. At the end of treatment: there was no direct effect of the newly used drugs on the cognitive function. After 3 months of treatment (SVR): there was significant improvement of the cognitive function. Conclusion: HCV eradication had beneficial effect on cognitive function. Sofosbuvir based treatment had no effect on cognitive function.
\end{abstract}

Keywords: Fibrosis - Hepatitis C Virus - Direct Acting Antiviral Drugs

\section{INTRODUCTION}

Hepatitis $\mathrm{C}$ virus (HCV) infection is considered a systemic disease because of involvement of other organs and tissues concomitantly with liver disease. According to WHO ${ }^{(I)}$ the three most common extrahepatic manifestations are depression (24\%), diabetes mellitus (15\%) and chronic renal disease (10\%). Among the extrahepatic manifestations, neuropsychiatric disorders have been reported in up to $50 \%$ of chronic HCV infected patients. Cognitive dysfunction, characterized by forgetfulness, attention and concentration difficulties, poor word recall, and delayed reaction times, has been documented in $13-50 \%$ of individuals with chronic $\mathrm{HCV}$ infection using comprehensive neuropsychological test panels (2). Although cognitive abnormalities are more common in individuals with advanced fibrosis and medical comorbidities, they are present even in the absence of advanced fibrosis and significant psychiatric and medical comorbidities ${ }^{(3)}$. The brain is a suitable site for $\mathrm{HCV}$ replication, where the virus may directly exert neurotoxicity; other mechanisms proposed to explain the pathogenesis of neuropsychiatric disorders in chronic HCV infection include derangement of metabolic pathways of infected cells, alterations in neurotransmitter circuits, autoimmune disorders, and cerebral or systemic inflammation. ${ }^{(4)}$. The most frequent psychiatric symptom reported in chronic $\mathrm{HCV}$ infection is fatigue mainly manifesting as physical and mental exhaustion, often in association with attention deficit and word-finding difficulty, depression, headache, osteoarticular pain, and sleep disturbances. Although insomnia has been reported in up to $60 \%$ of cases, ${ }^{(5)}$. Impact of HCV treatment and Sustained virus response on neuropsychiatric disorders: Although interferon- $\alpha$ treatment itself is known to possibly induce neuropsychiatric side effects, the drug ability to improve neurological and psychiatric symptoms in HCV patients achieving a sustained virological response (SVR) has been frequently reported in the literature ${ }^{(6)}$. Moreover, SVR has also been associated with improvement in cognitive function ${ }^{(7)}$. To date, studies addressing the issue of reversibility of cognitive deficits after HCV therapy are based on interferon treatment and to verify that improvement ${ }^{(8)}$.

\section{AIM OF THE WORK}

To evaluate the impact of $\mathrm{HCV}$ eradication using direct acting antiviral drugs (DAADs) on cognitive function among hepatitis $\mathrm{c}$ virus infected patients. 


\section{PATIENTS AND METHODS}

- Type of the study: A cohort observational study.

- Types of participants: Hepatitis c virus infected patients.

Duration of the study period: from September 2017 to June 2018.

62 patients fulfilling eligibility criteria were included in our study in the viral hepatitis unit of dar el shefaa hospital (one of the centers of national committee for control of viral hepatitis) and MASRI (faculty of Medicine Ain-Shams Research institute) unit at el-demerdash hospital. The study was approved by the Ethics Board of Ain Shams University and an informed written consent was taken from each participant in the study.

Inclusion Criteria: 1. Confirmed HCV infection by PCR. 2. Compensated liver disease (chronic hepatitis or child A cirrhotic). 3. >18 year. Exclusion criteria: 1. Patient child B or C. 2. $\mathrm{Plt}<50000$. 3. Uncontrolled systemic disease e.g., (DM, CKD HF) that may affect cognitive function. 4. Drug dependency or old cerebral stroke problem or any neurological and psychiatric symptoms. 5 . HCC or any extra hepatic tumors. 6. Pregnancy.

Methods: The patient received $\mathrm{HCV}$ treatment using direct acting anti-viral drugs according to the National Egyptian Protocol with pre, during and post treatment assessment of the following criteria. Pre-treatment assessment: 1. Full history taking: i.e., history of seizures, history of neurological and psychiatric disease, history of signs and symptoms of LCF, hematemesis, melena, weight loss, full drug history etc. 2. Full examination: general including signs of LCF and local abdominal examination including liver and spleen, full neurological examination. 3. Questionnaire assesses the cognitive and sleeping function of the patient: The Montreal Cognitive Assessment (MoCA test). 4. Liver profile including alanine aminotransferase (ALT), aspartate aminotransferase (AST), serum bilirubin, serum albumin and prothrombin time (Pt, INR). 5. Complete blood picture. 6. Alpha fetoprotein (AFP) level. 7. Renal function test. 8. HCV RNA PCR quantitative. 9. HBsAg. 10. FBS, (HbA1C for diabetic patient). 11. B HCG for female in child bearing period. 12. Abdominal ultrasound.
During the treatment every month: 1 . Full examination: general including signs of LCF and local abdominal examination including liver and spleen, neurological examination. 2. (ALT), (AST), serum bilirubin, serum albumin and prothrombin time (Pt). 3. Complete blood picture. 4. Renal function test.

Follow up of the patients at the end of treatment: 1. Full examination: general including signs of LCF and local abdominal examination including liver and spleen. 2. Questionnaire to assess the cognitive and sleep functions of the patient: The Montreal Cognitive Assessment (MoCA test). 3. (ALT), (AST), serum bilirubin, serum albumin and prothrombin time (Pt). 4. Complete blood picture. 5. Renal function test.

Follow up of patients after 3 months of treatment: 1. Full examination: general including signs of LCF and local abdominal examination including liver and spleen. 2. Questionnaire to assess the cognitive and sleep functions of the patient: The Montreal Cognitive Assessment (MoCA test). 3. HCV RNA PCR (quantitative).

- Types of outcome measures: Comparison between the 62 patients' own score before, after treatment and after 3 months of treatment.

- Ethical consideration: the study objectives was briefly and clearly described to all participants and an oral consent was obtained from all patients.

Statistical Analysis: Data were collected, revised, coded and entered to the Statistical Package for Social Science (IBM SPSS) version 23. The quantitative data were presented as mean, standard deviations and ranges when their distribution found parametric, median and inter quartile range (IQR) when their distribution found non parametric. Also qualitative variables were presented as number and percentages.

\section{RESULTS}

Table (1): Regimens of the treatment received by included patients

\begin{tabular}{|l|c|c|}
\hline \multicolumn{1}{|c|}{ Type of DAADs } & No. & \% \\
\hline Sofosbuvir / daclatasvir & 32 & $51.6 \%$ \\
\hline Sofosbuvir / daclatasvir /ribavirin & 24 & $38.7 \%$ \\
\hline sofosbuvir / simepriver & 6 & $9.7 \%$ \\
\hline Total & 62 & $100.0 \%$ \\
\hline
\end{tabular}


Table (2): Correlation of the MoCA scores at the baseline and at the end of treatment

\begin{tabular}{|l|l|c|c|c|c|c|}
\hline \multicolumn{2}{|l|}{} & Baseline & $\begin{array}{c}\text { End of } \\
\text { treat }\end{array}$ & $\begin{array}{c}\text { Test } \\
\text { valu } \\
\text { e }\end{array}$ & $\begin{array}{c}\text { P- } \\
\text { value }\end{array}$ & $\begin{array}{c}\text { Sig } \\
\cdot\end{array}$ \\
\cline { 1 - 7 } $\begin{array}{l}\text { MoC } \\
\text { A } \\
\text { test }\end{array}$ & $\begin{array}{l}\text { Mean } \pm S \\
\text { D }\end{array}$ & $\begin{array}{c}23.02 \pm 4.9 \\
6\end{array}$ & $23.73 \pm 4.78$ & 1.54 & 0.128 & NS \\
\cline { 2 - 4 } & $11-30$ & $13-30$ & 2 & & \\
\hline
\end{tabular}

There is no significant changes of the MoCA score at the end of treatment.

Table (3): MoCA score at the baseline and at 12 weeks after the end of treatment (SVR)

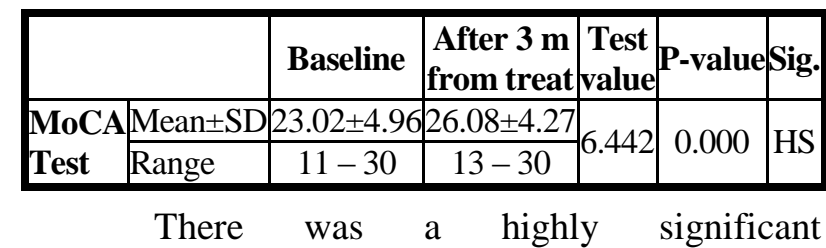

improvement in the results of MOCA test, after 12 weeks from the end of treatment indicating improvement of cognitive function after (SVR).

Table (4): Relation between the baseline FIB-4 score and the MoCA score.

\begin{tabular}{|c|c|c|c|c|c|c|}
\hline & & FIB- $4<3.25$ & FIB-4>3.25 & $\begin{array}{c}\text { Test } \\
\text { valu } \\
\text { e }\end{array}$ & $\begin{array}{c}\text { P- } \\
\text { value }\end{array}$ & Sig. \\
\hline $\begin{array}{l}\text { MoCA test } \\
\text { baseline }\end{array}$ & $\begin{array}{l}\text { Mean } \pm \\
\text { SD } \\
\text { Range }\end{array}$ & $\begin{array}{c}23.59 \pm \\
4.61 \\
11-30\end{array}$ & $\begin{array}{c}20.36 \pm \\
5.87 \\
12-29\end{array}$ & 2.003 & 0.050 & $\mathrm{~S}$ \\
\hline $\begin{array}{l}\text { MoCA test } \\
\text { after TT }\end{array}$ & $\begin{array}{l}\text { Mean } \pm \\
\text { SD } \\
\text { Range }\end{array}$ & $\begin{array}{c}24.10 \pm \\
4.68 \\
13-30 \\
\end{array}$ & $\begin{array}{c}22 \pm 5.06 \\
15-28\end{array}$ & 1.329 & 0.189 & NS \\
\hline $\begin{array}{l}\text { MoCA } \\
\text { after } \\
3 \mathrm{~m} \mathrm{TT}\end{array}$ & $\begin{array}{l}\text { Mean } \pm \\
\text { SD } \\
\text { Range }\end{array}$ & $\begin{array}{c}26.45 \pm \\
4.16 \\
13-30\end{array}$ & $\begin{array}{c}24.36 \pm \\
4.57 \\
16-30\end{array}$ & 1.483 & 0.143 & $\mathrm{NS}$ \\
\hline \multirow{2}{*}{$\begin{array}{l}\text { Repeated } \\
\text { measures } \\
\text { ANOVA }\end{array}$} & $\mathrm{F}$ & 18.015 & 15.600 & & & \\
\hline & $\mathrm{P}$-value & 0.000 & 0.000 & & & \\
\hline
\end{tabular}

Advanced fibrosis assessed by FIB-4 score above 3.25 had lower MoCA test score with a significant value indicating baseline cognitive impairment more with advanced fibrosis.

\section{DISCUSSION}

According to The EASL 2018, Hepatitis C virus (HCV) infection is a major cause of chronic liver disease, with approximately 71 million chronically infected individuals worldwide. Clinical care for patients with HCV-related liver disease has advanced considerably thanks to an enhanced understanding of the pathophysiology of the disease, and because of developments in diagnostic procedures and improvements in therapy and prevention. The primary goal of HCV therapy is to cure the infection, i.e. to achieve a sustained virological response (SVR) defined as undetectable HCV RNA 12 weeks (SVR12) or 24 weeks (SVR24) after treatment completion. In this study, we investigated the effect of an interferon-free treatment on cognitive function in $\mathrm{HCV}$ - infected patients by using valid scales in the Arabic language in an observational cohort study. The MoCA test was used to assess the cognitive function level among the included patients. Patients received treatment according to the Egyptian national committee of viral hepatitis and according to the availability of the drugs in the centers with percentage of $\mathbf{5 1 . 6 \%}$ for Sof and Dacla, $\mathbf{3 8 . 7 \%}$ for Sof, Dacla and Riba, $\mathbf{9 . 7 \%}$ for Sof and SIM. And all of the studied patients achieved the SVR12. Cognitive function: Our results showed that all the included patients had mild baseline cognitive impairment with mean MoCA test score was 23.02 \pm 4.96. This was consistent with that found by Cattie et al. ${ }^{(9)}$ who found the baseline of $22.5 \%$ of the cohort was Classified as globally neurocognitively impaired. Also Kleefeld et al. ${ }^{(8)}$ found that HCV-infected patients without comorbidities had significantly poorer Cognitive performance than controls. The cognitive function was re-evaluated by MoCA test at the end of treatment. The results revealed that during the treatment with the newly used DAADs there were no significant changes in the cognitive function with non-significant changes in mean from 23.02 \pm 4.96 to $23.73 \pm$ 4.78. This was consistent with Kleefeld et al. ${ }^{(8)}$ who found that at follow-up assessment of the patient treated with DAADs, the patients did not show cognitive decline in any domains compared to baseline during treatment. In contrast to data from the studies that discussed the effect of eradication of $\mathrm{HCV}$ using the interferon based therapy. Cattie et al. ${ }^{\left({ }^{9}\right)}$ found an increase of the patients that neurocognitively impaired from $22.5 \%$ of the cohort to $47.4 \%$ of patients by 10 weeks after initiating treatment. Nearly all of these patients $(42.9 \%)$ remained classified as globally impaired after 18 months, and mean global deficit scores remained significantly elevated relative to baseline levels at the final study visit. Mathew et $\boldsymbol{a l}^{\left({ }^{(10)}\right.}$ reviewed that Patients on IFN therapy should be monitored as IFN therapy may aggravate the 
HCV influenced neurological symptoms. The third evaluation of cognitive function was done 12 weeks after the end of treatment and revealed that MoCA test score significantly improved from 23.02 \pm 4.96 to $26.08 \pm$ 4.27. That indicates the effect of sustained virological response. Our results can be interpreted as sofosbuvir based treatment had no effect on cognitive performance while $\mathrm{HCV}$ eradication induce that improvement. These results are compatible with other results from previous studies. Kraus et al. ${ }^{(1)}$ reported successful HCV eradication with peginterferon and ribavirin was associated with improved attention, vigilance, and working memory, while virological non-responders showed no such improvements. While Onali et al. (12) carried out a single centre prospective study on 32 co-infected HIV-HCV patients and treated with DAADs to examine changes in neuro-cognitive status of HIV-HCVco-infected patients treated with DAADs, MoCA test was used. It was carried out at baseline (before starting therapy) and 6 months after the end of treatment. the results suggested that co-infected HCV-HIV patients who achieved SVR improve neurocognitive functions, such as execution and memory, emphasizing the importance of treatment with antivirals direct acting not only for the eradication of HCV (SVR $100 \%$ in our treated patients), but also in the improving of neuro-cognitive disorders. This conclusion agrees with that indicated by Byrnes et al. ${ }^{(13)}$ who reported that there is improvement in cerebral immune activation associated with $\mathrm{HCV}$ clearance, using brain proton MRS and cognitive assessments before, during and after interferonbased therapy, showed significant CNS metabolic changes towards normal levels in those patients who had an SVR. Also Alsop et al. ${ }^{(14)}$ reported normalization of cerebral $\mathrm{N}$-acetyl aspartate using MRS, in patients with viral suppression with ledipasvir + sofosbuvir, which was interpreted as recovery of neuronal dysfunction.

\section{CONCLUSION}

HCV eradication had beneficial effect on cognitive function. Sofosbuvir based treatment had no effect on cognitive function. Advanced fibrosis (as assessed by FIB-4) was related to more advanced baseline cognitive impairment.

\section{CONFLICTS OF INTEREST}

There are no conflicts of interest.

\section{REFERENCES}

1. World Health Organization (2018): Guidelines for the care and treatment of persons diagnosed with chronic hepatitis $\mathrm{C}$ virus infection. www.who.int/hepatitis/ publications/hepatitis-c-guidelines-2018/en/

2. Fontana R, Bieliauskas L, Lindsay $\mathrm{K}$ et al. (2007): HALT-C Trial Group. Cognitive function does not worsen during pegylated IFN and ribavirin re-treatment of chronic hepatitis C. Hepatology, 45: 1154-1163.

3. Forton DM, Thomas HC, Murphy CA et al. (2002): Hepatitis $\mathrm{C}$ and cognitive impairment in a cohort of patients with mild liver disease. Hepatology, 35(2): 433-9.

4. Adinolfi LE, Nevola R, Lus G et al. (2015): Chronic hepatitis $C$ virus infection and neurological and psychiatric disorders: An overview. World Journal of Gastroenterology: WJG., 21(8): 2269-2280.

5. Ashrafi M, Modabbernia A, Dalir $M$ et al. (2012): Predictors of mental and physical health in non-cirrhotic patients with viral hepatitis: a case control study. J Psychosom Res., 73: 218-224.

6. Sarkar S, Jiang Z, Evon DM et al. (2012): Fatigue before, during and after antiviral therapy of chronic hepatitis $\mathrm{C}$ : results from the Virahep-C study. J Hepatol., 57: 946952.

7. Thein HH, Maruff P, Krahn MD et al. (2007): Improved cognitive function as a consequence of hepatitis $\mathrm{C}$ virus treatment. HIV Med., 8: 520-528.

8. Kleefeld $\mathrm{F}$, Heller $\mathrm{S}$, Jessen $\mathrm{H}$ et al. (2016): Effect of interferon-free therapy on cognition in HCV and HCV/HIV infection: a pilot study. Neurology, 10-212.

9. Cattie JE, Letendre SL, Woods SP et al. (2014): Persistent neurocognitive decline in a clinic sample of hepatitis $C$ virus-infected persons receiving interferon and ribavirin treatment. J Neurovirol., 20: 561-570. 
10. Mathew S, Faheem M, Ibrahim SM et al. (2016): Hepatitis $C$ virus and neurological damage. World Journal of Hepatology, 8(12): 545.

11. Kraus MR, Schafer A, Teuber G et al. (2013): Improvement of neurocognitive function in responders to an antiviral therapy for chronic hepatitis C. Hepatology, 58(2): 497-504.

12. Onali S, Pacini DG, Balestrieri C et al. (2017): Evaluation of Mild Cognitive Dysfunction by Montreal Cognitive Assessment Test in Patients with Chronic HCV Infection Treated with Direct Antiviral Agents. Digestive and Liver Disease, 49(1): 24-29.
13. Byrnes V, Miller A, Lowry $D$ et al. (2012): Effects of anti-viral therapy and $\mathrm{HCV}$ clearance on cerebral metabolism and cognition. J Hepatol., 56(3): 549-56.

14. Alsop D, Younossi Z, Stepanova $M$ et al. (2014): Cerebral MR spectroscopy and patient-reported mental health outcomes in hepatitis $\mathrm{C}$ genotype 1 naive patients treated with ledipasvir and sofosbuvir. Hepatology, 60: 221A. 